\title{
Design and Implementation of Micro Mobile MPLS for NS-2
}

\author{
Tubtim Sanguanwongthong \\ School of Engineering and Technology \\ Asian Institute of Technology \\ Klong Luang, Pathumthani, 12120, Thailand \\ st102152@ait.ac.th
}

\author{
Prawit Chumchu \\ Information and Communication Engineering (ICE) \\ Mahanakorn University of Technology \\ Nong Chok, Bangkok, 10530, Thailand \\ prawit@mut.ac.th
}

\begin{abstract}
In this paper, we extend NS-2 to simulate Micro-Mobile MPLS frameworks. The extension supports most of the important mechanisms used in the literature. The simulator is based on IEEE 802.11. The important mechanisms for IEEE 802.11 in our module are multi-channel, directional antennas, multiple interfaces, L2-handoff, L2-trigger, bicasting, pre-established LSP, and buffering mechanism. MPLS modules used in this simulator is based on MPLS module (MNS) [8]-[10]. This simulator could be configured to assess performance of Mobile MPLS protocols such as micro mobility-enabled multiprotocol label switching (MMPLS) [21], fast handoff (FH) and master forwarding chain (MFC)-Micro Mobile MPLS [2], [23] and MiM-MPLS [30]. The simulator is validated for each mechanism. The validation results follow to the theory.
\end{abstract}

\section{Categories and Subject Descriptors}

C.2.1 [Computer-Communication Networks]: Network Architecture and Design; I.6.5 [Simulation and Modeling]: Model Development

\section{General Terms}

Performance, Design, Experimentation

\section{Keywords}

Mobile MPLS, micro-mobility, NS-2, module

\section{INTRODUCTION}

Future wireless network is evolving into all IP-based architecture with efficient mobility support and end-to-end Quality of Service (QoS). To support mobility based IP and seamless roaming to mobile applications/devices (e.g. mobile IP phone, pocket PC phone, etc.) in the Internet, Mobile IP, a standard proposed by the Internet Engineering Task Force (IETF), is the only current means to serve that. However, there are several drawbacks such as long handoff latency, transient packet loss, scalability, and high signaling load [1], [18]. Owing to the notable benefits of MultiProtocol

Permission to make digital or hard copies of all or part of this work for personal or classroom use is granted without fee provided that copies are not made or distributed for profit or commercial advantage and that copies bear this notice and the full citation on the first page. To copy otherwise, or republish, to post on servers or to redistribute to lists, requires prior specific permission and/or a fee.

WNS2 2008, October 23, 2008, Athens, GREECE.

Copyright (C) 2008 ICST ISBN \# 978-963-9799-31-8.
Label Switching (MPLS) in terms of QoS, Traffic Engineering (TE), and support of advanced IP services including differentiated services (DiffServ), the research on the integration of the Mobile IP and MPLS protocols was thus inspired to use this technology in the wireless infrastructure [2], [23]. Additionally, this technology gives telecommunication service provider major economical advantages of providing mobility to existing legacy network and new services to users immediately besides allowing a smooth upgrade of the networks [3].

Nonetheless, Mobile MPLS is generally only suited to macromobility which concerns the scalability as a main issue while it does not efficiently support micro-mobility which concerns the mobility as a main issue [4]. Consequently, most researches in this area aim Micro Mobile MPLS to solve the mobility problem [2], [4]- [5], [13]-[17] [21] and [23]. Furthermore, they have used the simulators to verify their proposals such as NS-2 [5], OPNET [6], etc. and they might perform performance analysis as well while some papers did not specify which simulator they used or how to model simulation. Additionally, most works did not provide how to model the simulation or how to design and develop the simulation model. Also, few works [18], [21] only proposed their idea without implementation and simulation.

For the reason that most studies have been based on simulation or verified their work using NS-2 [19], NS-2 therefore becomes a de facto standard for the simulation of networking research used by academia and industry [19] and [20]. In this paper, we consequently focus on how to model Micro Mobile MPLS module for NS-2.

In view of this, Langar et al. [2], [23] proposed two solutions, fast handoff (FH) and master forwarding chain (MFC)-Micro Mobile MPLS, to enhance handoff support in micro-mobility MPLS, and verified their proposal by performing performance analysis and simulation using NS-2 without giving in detail about how to develop the simulation module.

Estrela et al. [3] proposed a mobility solution, an enhanced version of terminal independent Mobile IP (eTIMIP), which provides both network and terminal independent mobile architectures based on the use of an overlay micro-mobility architecture. They evaluated their proposal by performing simulation using ns-2.26 and CIMS v1.0 mobility suite [7] without giving in detail about the simulation module, too.

Langar et al. [4] reviewed some of the most prominent micromobility protocols: cellular IP, handoff-aware wireless access Internet infrastructure (HAWAII), Mobile IP regional registration 
protocol (MIP-RR), overview of Mobile MPLS, and Micro Mobile MPLS. Additionally, there is only a brief overview of the simulation module about MPLS hierarchical node architecture. An updated version of IP micro-mobility module CIMS v1.0 [7] contributed by Columbia University and the MPLS module (MNS) [8] contributed by Gaeil Ahn [8], [9] and [10] were used.

Ganchev [12] proposed a schematic structure of the integration of wireless base station node and MPLS node based on ns-2.1b8a and MNS-v2.0 for ns-2.1b8a [11] contributed by Christian Glomb. Nevertheless, it did not have the validation test in the proposal.

Fowler et al. [13] proposed a fast handoff approach over Micro Mobile MPLS to provide lower end-to-end delays and evaluated performance by using NS-2. Also, they did not provide in detail about simulation module.

Papers in [14]-[17] proposed solutions to ameliorate handoff in Micro Mobile MPLS and performed simulations using NS-2. They used ns-2.1b6 with MNS extension [8]-[10] and CMIS module [7]; however, they did not provide in detail how to integrate these modules as well.

To sum up, there have been, to the best of our knowledge, very few researches [4] and [12] described the Mobile MPLS module for NS-2 and these work provided a very brief detailed description of the module. Furthermore, ns-2.33, the current NS-2 release (March 31, 2008), still does not have the feature of Mobile MPLS. Owing to these reasons, in this paper, we design and implement the Mobile MPLS module with most essential features for NS-2. The important mechanisms are multi-channel support used in IEEE 802.11, directional antennas, multiple interfaces, L2-hand over, L2-trigger, bicasting, pre-established LSP, and buffering mechanism.

The remainder of this paper is organized as follows. Section 2 provides a brief review of Micro Mobile MPLS. Our proposed design and implementation of Mobile MPLS module is discussed in section 3. A description of the tests performed for a functional validation of the developed module is provided in section 4. Finally, section 5 concludes the paper with some final remarks.

\section{AN OVERVIEW OF MICRO MOBILE MPLS}

Mobile MPLS is a scheme that integrates the Mobile IP and MPLS protocols in order that MPLS improves the performance of Mobile IP in terms of data forwarding at label switching, QoS (Quality of Service), TE (Traffic Engineering), and support of advanced IP services; in return, Mobile IP provides mobility to MPLS [2], [4]- [5], [13]-[17] and [23]. Since several research [2], [4]- [5], [13]-[17] [21] and [23] in this area proposed solutions for mobility problem, we thus propose the Micro Mobile MPLS module for NS-e in this paper. Based on a practical approach, Micro Mobile MPLS is usually described by using hierarchical architecture [2], [4]- [5], [13]-[17] [21] and [23]. A typical architecture of Micro Mobile MPLS is illustrated in Figure 1. The network architecture is based on a two-level hierarchy. At the higher level is the Label edge router Gateway (LG) that performs the role of an edge Label Switch Router (LSR) filtering between intraand inter- domain signaling. At the other level is the Label edge router/Foreign Agent (LFA) connected to several access points
(APs) that offer link-layer connectivity as mobile node (MN) connected to APs moves inside the Micro Mobile MPLS access network.

The following subsections review some of the most prominent micro-mobility protocols: micro mobility-enabled multiprotocol label switching (M-MPLS) [21], (FH and MFC)-Micro Mobile MPLS [2][23], and our framework named Micro-Mobility MPLS (MiM-MPLS) [30]. Note that our proposed NS-2 module is not only implemented to support most mechanisms of the Micro Mobile MPLS frameworks described in this section such as L2 handoff, bicasting, L2 trigger, buffering mechanism, etc. but also designed to support most essential mechanisms of Micro Mobile MPLS protocols.

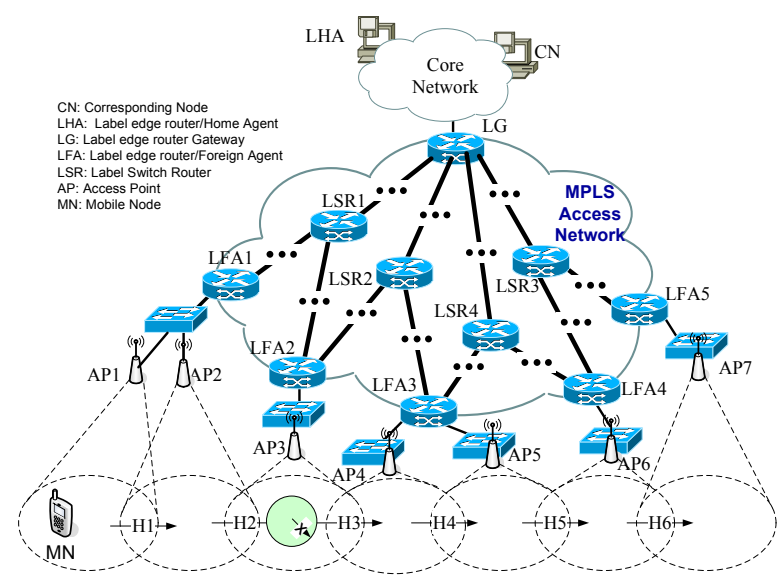

Figure 1. Architecture of a Micro Mobile MPLS Wireless Access Network

\subsection{M-MPLS}

M-MPLS [21], the integration of MPLS and hierarchical mobile IPv6 (HMIPv6), is proposed in two modes of operation: an overlay and an integrated framework. In an overlay framework, MPLS and HMIP operate independently of each other (i.e., without having common process, tables, or signaling). This causes the inefficiency of changing layers because MPLS modules do not communicate directly with Mobile IP. On the other hand, in an integrated framework related functions are merged; as a result, network operations are faster and more optimized, which gives better network utilization and a reduction in processing time. In other words, the integrated framework is the optimized overlay framework which involves two major components, node architecture for MPLS supporting mobility and rerouting for handling handoffs and mobility-triggered mechanisms of MPLS.

The network architecture is based on hierarchy similar to Figure 1. In [21], radio access router (RAR, LFA in Figure 1) is an edge LSR which connects intermediate LSRs and provides connectivity to mobile terminals. The mobility anchor point (MAP, LG in Figure 1), another edge LSR, connects to other subnets. A care of address $(\mathrm{CoA})$ is used as a forwarding equivalence class (FEC) in label forwarding information base (LFIB). The packet is encapsulated along HA to RAR tunnel. Additionally, the encapsulation header has CoA which is the destination address (i.e., FEC). To have the common operation of MPLS and MIP in the same layer, 
LFIB is modified by adding a new field. This field contains a pointer to another entry in the same table.

When CN sends a message to MN, a LSP is established along CN's RAR-HA-MAP-MN's RAR path. That is, the message is sent along the LSP to MN. During handoff, the MAP as an ingress LSR is informed that the path needs to be changed. It then sends out an LSP modification message and waits for a new label to return for use in a new LSP.

\subsection{FH-Micro Mobile MPLS}

FH-Micro Mobile MPLS [2][23] aims to reduce service disruption during handoff by pre-establishing an LSP before the MN moves into a new subnet. The network architecture of the proposal is described by using a hierarchy similar to Figure 1. The Label Edge Router Gateway (LERG, LG in Figure 1), an edge LSR, is capable of filtering packets between different domains and the Label Edge Router/Foreign Agents (LER/FA, LFA in Figure 1), another edge LSR, connects several base stations (BSs). There are two types of LSP defined in this framework: active LSP and passive LSP. The active LSP is the current LSP used to transfer data between LERG and the current serving LER/FA. The other LSP, a passive LSP, is a pre-established LSP between LERG and the next destined LER/FA which is not currently used until its activation.

When MN enters an overlapped area of the boundary cells of two subnets, MN receives an L2 trigger for an imminent L2 handoff. Then, MN will send a handoff signaling message to notify the current LER/FA which relays the message to LERG for the possible handoff. At the same time of LERG receiving the message, the LERG immediately initiates the LSP procedure set up with the new LER/FA before the L3 handoff occurs. That is, a passive LSP between the LERG and the new destined LER/FA will be preestablished. At that time, the current LER/FA informs the MN about the new LER/FA's IP address. So that, as soon as MN moves to the new subnet, it informs its arrival and starts the registration process with the LERG. The pre-established LSP will be thus activated and traffic will be delivered through the activated LSP.

\subsection{MFC-Micro Mobile MPLS}

MFC-Micro Mobile MPLS [2][23], described by the same network architecture as FH-Micro Mobile MPLS in subsection 2.2, aims to handle local mobility efficiently. This framework is based on the forwarding chain concept. In other words, there is a master LER/FA so that MN every time moving to the new subnet performs a local registration with the master LER/FA only instead of the LERG. To avoid a long forwarding chain, a threshold on its length is set. When the length of forwarding chain reaches the threshold, the MN will register to the LERG and the MN forwarding chain will be renewed. That is, the new visited LER/FA becomes the new master LER/FA. Also as in subsection 2.2, the L2 trigger is used in this framework to inform $\mathrm{MN}$ of an imminent L2 handoff.

\subsection{MiM-MPLS}

The network architecture of MiM-MPLS [30] is based on a hierarchical architecture as shown in Figure 1. At the time of network initialization, LSPs are pre-established in MiM-MPLS between LFAs and LG. Besides, MiM-MPLS has features on bicasting, buffering mechanism and pre-registration mechanism these for ameliorating the handoff performance of MiM-MPLS.
When $\mathrm{CN}$ sends a message to $\mathrm{MN}$, the message is sent along the pre-established LSP(s) to MN. At the time, MN reaches the boundary of an effective area of the current AP (X in Figure 1), it receives a L2 beacon from the possible new AP. On the assumption that a link layer movement detection scheme is in MiMMPLS to predict the possible MN's next location. The MN then performs the pre-registration mechanism with the new LFA. At the same time, the old LFA starts buffering in-flight packets. When MN reaches the boundary of an overlapped area, the MN receives the $\mathrm{L} 2$ trigger and sends a Mobile IP moving message to the old LFA. The old LFA sends the reply message back to the $\mathrm{MN}$ and L2 handoff occurs. The MN then sends the Mobile IP registration message with that new LFA and the new LFA relays the registration message to LG. At that time, the old LFA stops buffing in-flight packets and then forwards buffered in-flight packets destined for the $\mathrm{MN}$ toward the new subnet along the preestablished LSPs. So in this case, the MN may receive packets from the old LFA via the new LFA before the L3 handoff completes (i.e., before $\mathrm{MN}$ receiving the registration reply from the LG).

\section{MICRO MOBILE MPLS MODULE FOR} NS-2

In this section, we present Micro Mobile MPLS Simulator. We do not implement a specified Mobile MPLS protocol. This is because there are many Mobile MPLS protocols. Currently, they are not standardized. Therefore, we implement and design generic frameworks with most important mechanisms that does not support in NS-2. The essential features are multi-channel simulation, multiple-interfaces, bi-directional antennas, L2-trigger, bicasting, buffering and pre-established LSP. To test all, we choose IEEE 802.11 as multiple access protocol. This is because WLANs have been extremely deployed in public and residential places for various wireless applications due to low deployment cost and broadband bandwidth capability.

We describe signaling and data delivery for generic Micro Mobile MPLS that will be used to implement in NS-2. The network scenario is shown in Figure 1. The signaling signals are shown in Figures 2, 3, 4, and 5. The signaling signals are classified based on types of handoff. Handoff considered in our scheme is classified into 2 types: Intra-LFA (L2-Intra-LFA and L3-Intra-LFA) and Inter-LFA. When the MN moves between two APs managed by the same LFA called an Intra-LFA handoff, but different LFAs called an Inter-LFA. In Figure 1, handoff H1 is L2-Intra-LFA handoff and handoff $\mathrm{H} 4$ is L3-Intra-LFA handoff while handoffs $\mathrm{H} 2, \mathrm{H} 3, \mathrm{H} 5$, and $\mathrm{H} 6$ are Inter-LFA handoff.

\subsection{Network Initialization}

The extended simulator could be configured to support preestablished LSPs between LSPs and LGs. The selection of LGs is performed by LFAs to balance traffic and QoS control. Currently, the selection could be done by manual configuration. In addition, to provide different class of service, the simulator could be configured to support the DS-TE by using RSVP/TE [26].

\subsection{Registration Procedure}

The registration of the extended simulator could be described as follows. The steps of initial registration for a $\mathrm{MN}$ are presented in Figure 2. When $\mathrm{MN}$ moves for the first time into a foreign domain, it obtains a Regional Care-of-Address (RCoA) and Care of 
Address (CoA) broadcast periodically by its LFA1. Then, the MN sends a Mobile IP registration request message (Reg. Req. in Figure 2) including the RCoA and its home address to LFA1. When the message reaches the LFA1, the LFA1 records the MN home address in its routing table. After that, LFA1 relays the message to the LG along the pre-established LSP in the network initialization phase.

When the LG gets the Reg. Req and knows RCoA which corresponds to the IP address of the current LFA1, it sends a Reg. Req. to the HA of the MN. The LG uses its IP address as the care-of address $(\mathrm{CoA})$ to perform the global registration for inter-domain mobility. Once the simulator is configured to test Pure Mobile MPLS, one entry of which FEC is MN address is added in LFIB. The outgoing label and outgoing interface are the same as the entry of RCoA. At the end, the LG forwards the registration reply (Reg. Reply) message sent from the LHA to the MN along the pre-established LSP via LFA1.

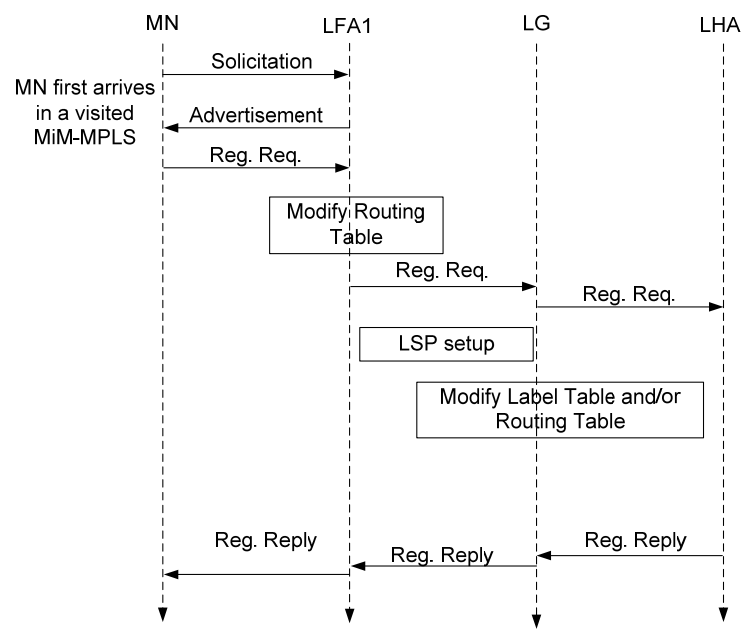

Figure 2. Registration

\subsection{Intra-LFA Handoff}

\subsubsection{L2-Intra-LFA Handoff}

According to Figure 1, the L2-Intra-LFA handoff is presented in H1. Additionally, Figure 3, which NAP stands for new AP and OAP stands for OAP, describes the procedure of L2-Intra-LFA handoff. As L2 current signal strength is lower than an unacceptable level or the number of lost ACK is greater than an unacceptable level, the MN sends a movement signaling message to the current LFA, which initiates the buffering mechanism and stores in-flight packets. After that the MN will scan the air interface for a new AP. If it finds a good one, it will register at layer 2 with that AP. Then, the MN either waits for a Mobile IP Advertisement message sent from the LFA or it will issue a Mobile IP Solicitation message. In any case, the MN examines the prefix of LFA's IP address. If this prefix is the same as before handoff, it means that the MN is under the same IP subnet. Therefore, the MN will issue a local interface-update message in the subnet to which the $\mathrm{MN}$ belongs, so that all stations in the same subnet, especially the current LFA, update their Address Resolution Protocol cache. In this case, the current LFA will stop the buffering mechanism and forward in-flight packets destined for the MN toward the new AP.

\subsubsection{L3-Intra-LFA Handoff}

As shown in Figure 1, the L3-Intra-LFA handoff is presented in H4. Also, the procedure of L3-Intra-LFA handoff is described in Figure 4. Similarly to L2-Intra-LFA handoff, the L3-Intra-LFA handoff is serviced by the same LFA. However, this handoff occurs between the two different subnet APs. Consequently after the $\mathrm{MN}$ receives a Mobile IP advertisement message, the MN examines the prefix of LFA's IP address. If the prefix is different before the handoff but the same current RCoA, it means that the MN is still under the same LFA but the two different subnet APs. Then, the MN will send a Reg. Req. to the LFA. The LFA updates its routing table and send Reg. Reply to MN. At the same time, the LFA will stop the buffering mechanism and forward in-flight packets destined for the MN toward the new AP.

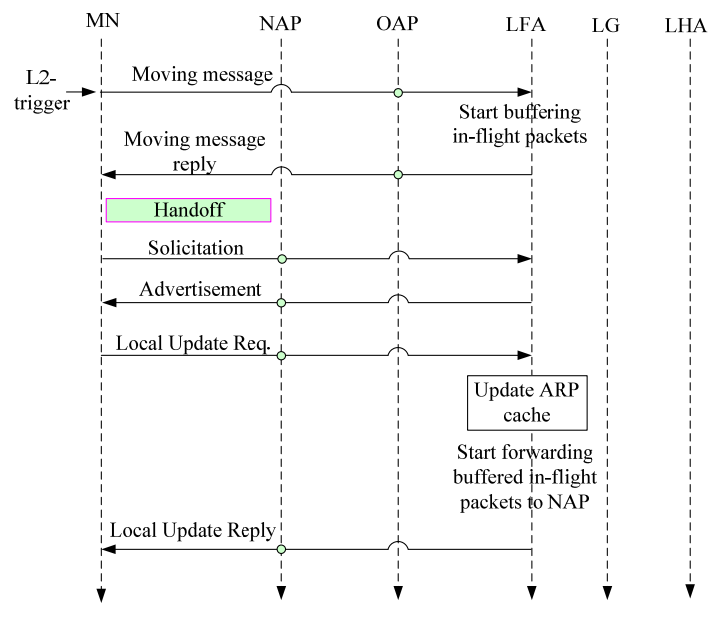

Figure 3. L2 Intra-LFA Handoff

\subsection{Inter-LFA Handoff}

As illustrated in Figure 1, the Inter-LFA handoff is presented in $\mathrm{H} 2, \mathrm{H} 3, \mathrm{H} 5$, and H6. The procedure of Inter-LFA handoff is described in Figure 5. When MN receives the L2 trigger, it sends a movement message to current LFA (i.e., old LFA or OLFA) via OAP. Then, the OLFA starts buffering in-flight packets, and sends a moving message reply back to MN. In addition, L2-triger could help to do bicasting to reduce handoff loss. The signaling for pre-registration is shown in Figure 5. After MN sends a solicitation message to NLFA and receives an advertisement message from NLFA, the MN examines the prefix of NLFA's IP address from the advertisement message. If the prefix differs from the current LFA, it means that the MN has moved to NLFA. Then, the MN sends a Reg. Req to the NLFA. The NLFA updates its routing table. At the same time, the MN sends a handoff notification message to the OLFA via NLFA along pre-established LSPs. Upon receiving the handoff notification message, the OLFA stops the buffering mechanism and forwards in-flight packets destined for the MN toward the new subnet along the pre-established LSPs. So in this case, the MN may receive packets form OLFA via NLFA before the L3 handoff completes (i.e., before receiving a Reg. Reply from the LG). After receiving Reg. Req message, the LG will stop bicasting and send Reg. Reply message to the $\mathrm{MH}$ via the NLFA. 


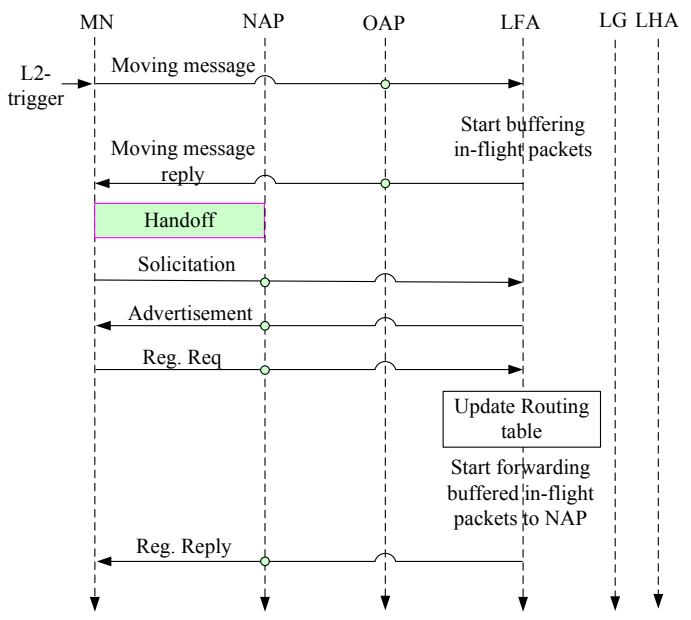

Figure 4. L3-Intra-LFA Handoff

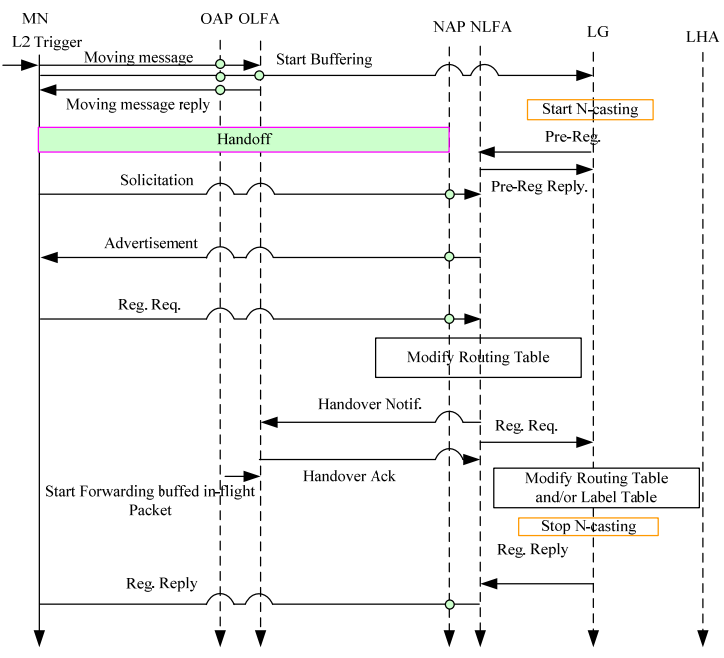

Figure 5. L3-Inter-LFA Handoff

\subsection{Data Delivery}

The data delivery from LHA to LG could be IP tunneling such as in [4], IP tunneling and MPLS delivering such as in [21], and MPLS delivering such as in [22]. Similarly, the data delivery from LG to LFA could be IP tunneling and MPLS delivering such as in [30] and [21], and pure Mobile MPLS delivering such as in [4].

\subsection{L2 handoff}

According to section 1, L2 protocol used in our work is IEEE 802.11. IEEE 802.11-based WLANs specification allows for two operating modes: ad hoc mode and infrastructure mode. In Ad hoc mode, two or more 802.11-based wireless clients can recognize each other or one another communication without the need of a AP. In infrastructure mode, 802.11-based wireless clients are associated with an AP that bridges with wired networks i.e., Ethernet. In this work we focus on the latter mode. Clients can move from an associated AP to neighbor APs. This mobility needs a low latency handoff algorithm to keep seamless connection. The complete handoff process in the IEEE 802.11 standard could be divided into three distinct phase: scanning or discovery, authentication and re-association. Figure 6 shows the detail of the handoff procedures. The handoff process begins after service degrades below an acceptable threshold such as the signal-tonoise ratio of the current link, received signal strength of current link, or packet losses of current communication. Figure 7 illustrates an example of the received signal strength at the time of handoff. It could be seen from the figure that point $\mathrm{A}$ is the cell search threshold. Point B and C are the time to trigger L3 to perform special mechanisms such as bicasting, buffering. Then the client continuously scans all channels by varying the transceiver frequency. Then, it selects the best AP using service metrics (i.e., number of lost acknowledgement, signal strength). It then sends an authentication request to the new AP and waits for reauthentication response. The authentication phase could be opensystem authentication, shared-key authentication or IEEE 802.11i authentication. After receiving the re-authentication response, it starts re-associate process by sending Re-associated request. The handoff process is completed after receiving re-association response.

There are currently two kinds of channel scanning methods in IEEE 802.11 standard: passive and active. For example, selective channel scanning using neighbor graph, selective channel scanning using sensor Networks and synchronized beacon scanning (SyncScan) [28].

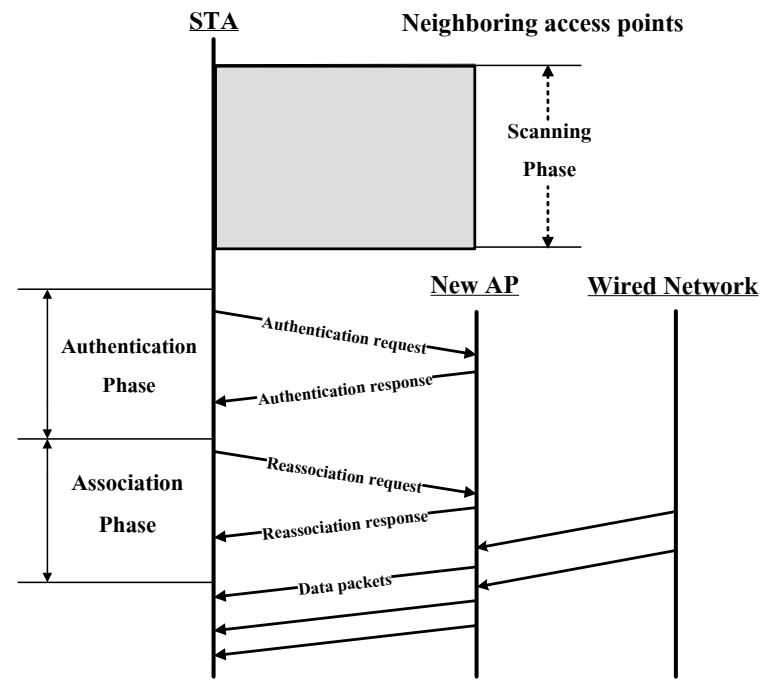

Figure 6. IEEE 802.11 Handoff Algorithm

\subsubsection{Passive Scanning}

On the assumption that client associates with AP1 in channel 1, If it needs handoff because service degrades below an acceptable threshold, it will switch operating frequency to listen for all other frequencies in lists for example channels 2 to 11 . After scanning all channels, it decides to associate with the AP whose link quality is the best. 


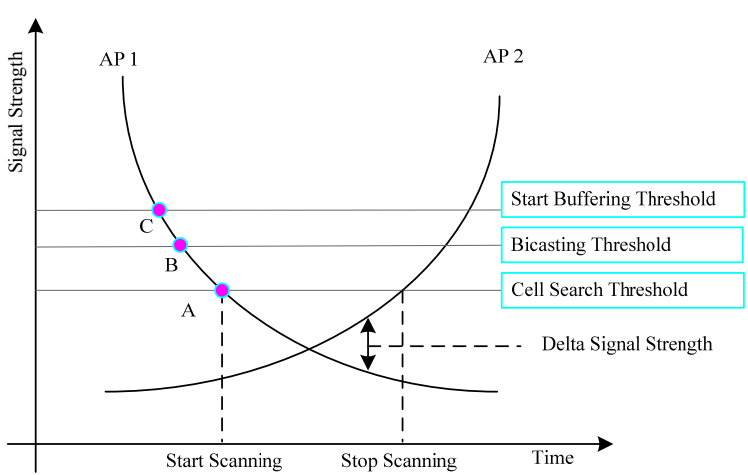

Figure 7. Example of the Received Signal Strength at the time of Handoff

\subsubsection{Active Scanning}

Active scanning performs as follows. When needed handoff, the wireless client broadcasts a probe request packet on a possible channel and waits a probe response. If it could not receive any probe response after MinChannel Time, it will start to probe for the next channel in lists. If it receives a probe response in MinChannel Time, it will wait to collect for possible probe responses. It will request for all channels except the associated AP's channel. After requesting all channels, it designs to associate the AP whose link quality is the best.

In simulation section, we use received signal strength at L2 to trigger L2 to perform bicasting, buffering.

\subsection{Node Architecture}

The module has been implemented by extending NS-2 [5]. To support hierarchy address and LSR capable of mobility, MPLS node is extended to be LG (Figure 8), LFA or LHA (Figure 9), and MN (Figure 10).

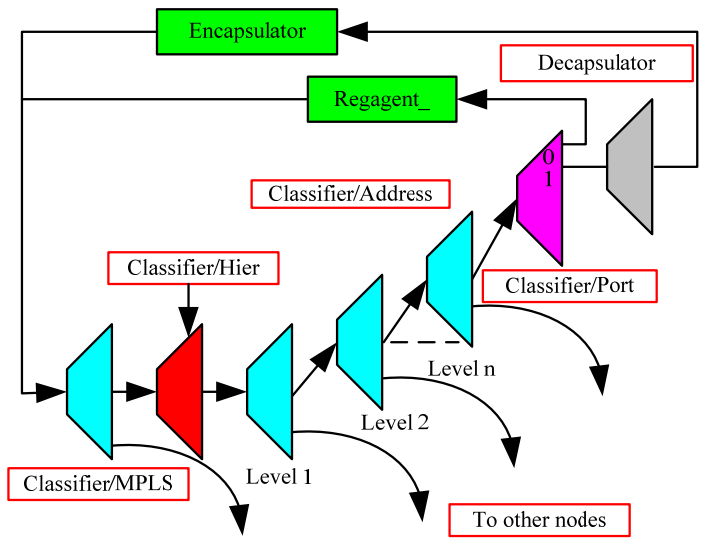

Figure 8. LG Node

In Figure 8, 'Encapsulator', 'Regagent_' (i.e., registration agent), and 'Decapsulator' are inserted into a MPLS node of MNS for making the LG node. 'Encapsulator' is to encapsulate packets between its LG and LFA. 'Regagent_' is to support the registra- tion process of the mobile node. 'Decapsulator' is to decapsulate packets sent to $\mathrm{MN}$ via $\mathrm{LG}$.

The LFA node and the LHA node have the same node architecture as illustrated in Figure 9. The dash line from 'Classifier/Hier' to 'Ragent_' is referred to routes to visiting MNs. Each route is created when a MN registers to a LFA of micro-mobility frameworks using pure Mobile MPLS (i.e., without IP tunneling). We could not add routes to Classifier/Addresses as normal because each 'Classifier/Address' independently performs routing decision. It will make wrong decision if we add route as normal.

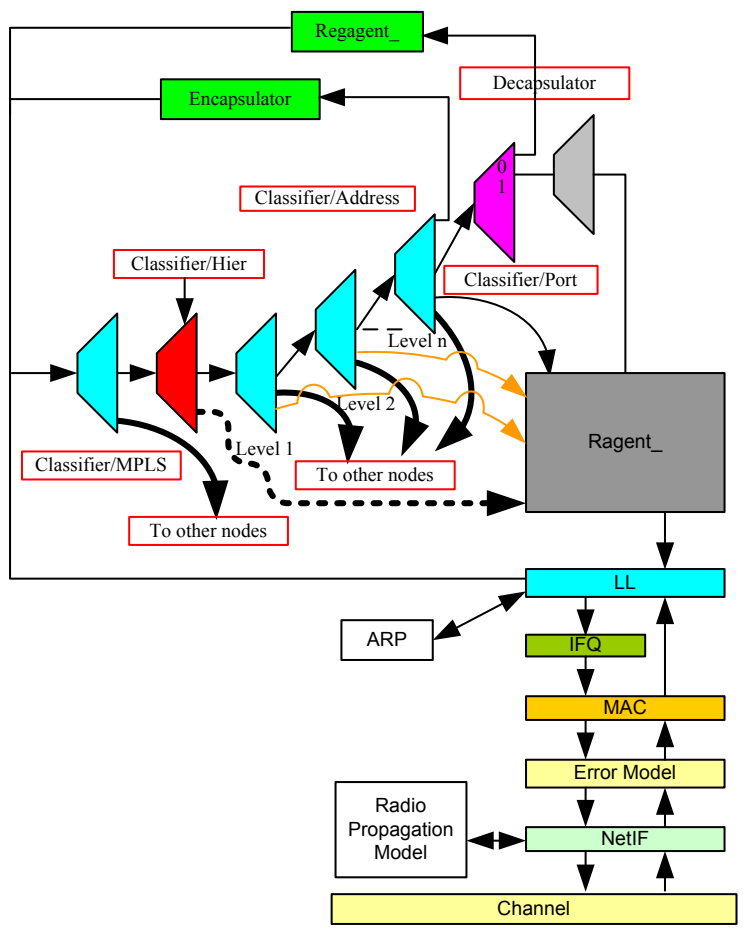

Figure 9. LFA/LHA Node

The architecture of MN node is shown in Figure 10. This node is extended from original NS-2 to support multi-channel, multiple interfaces, direction antennas and different types of L2 handoff such as IEEE 802.11 passive scanning [27], IEEE 802.11 active scanning [27], SyncScan [27] and AsyncScan [29]. 


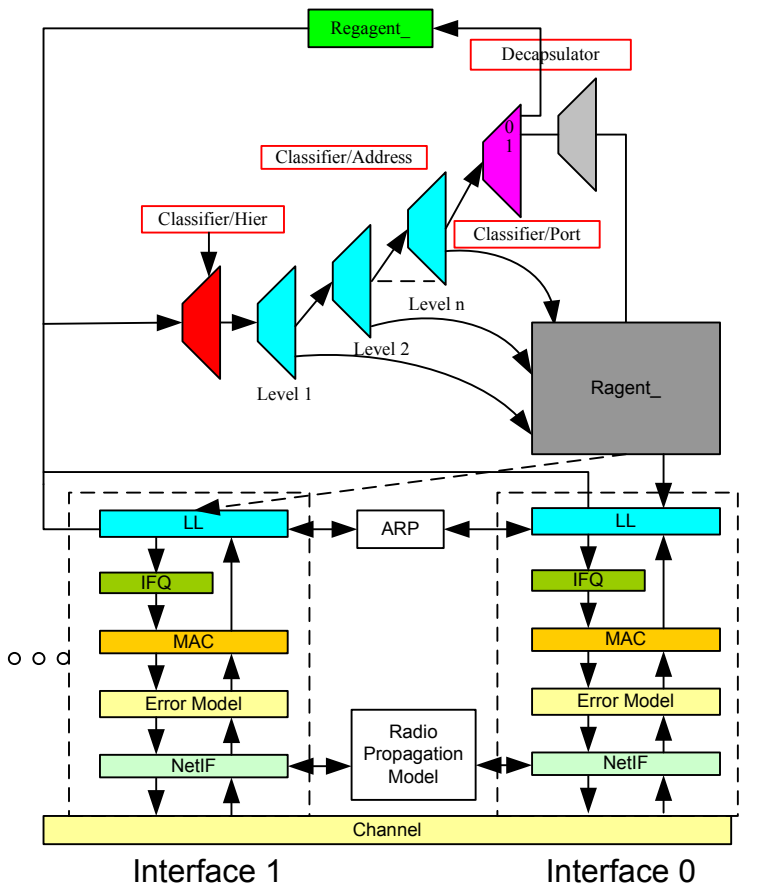

Figure 10. MN Node with 2 interfaces

\section{FUNCTIONAL VALIDATION TESTS}

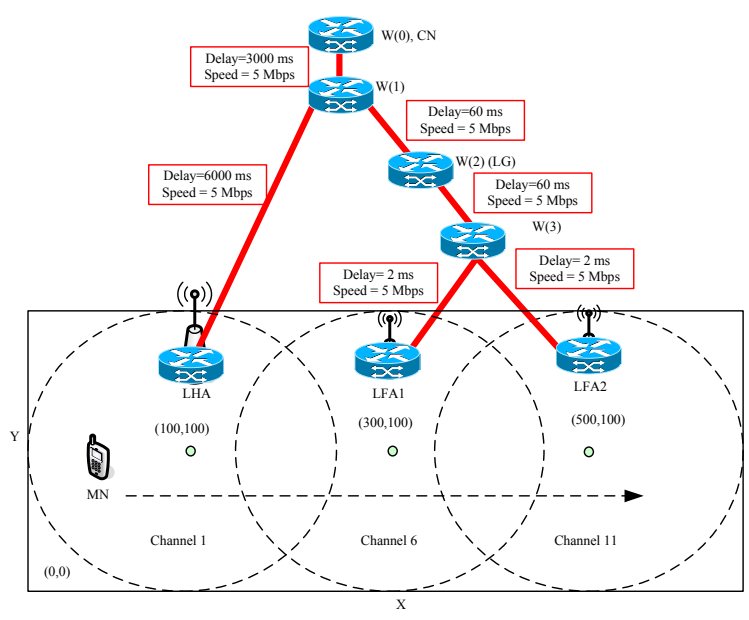

Figure 11. Simulation Scenario

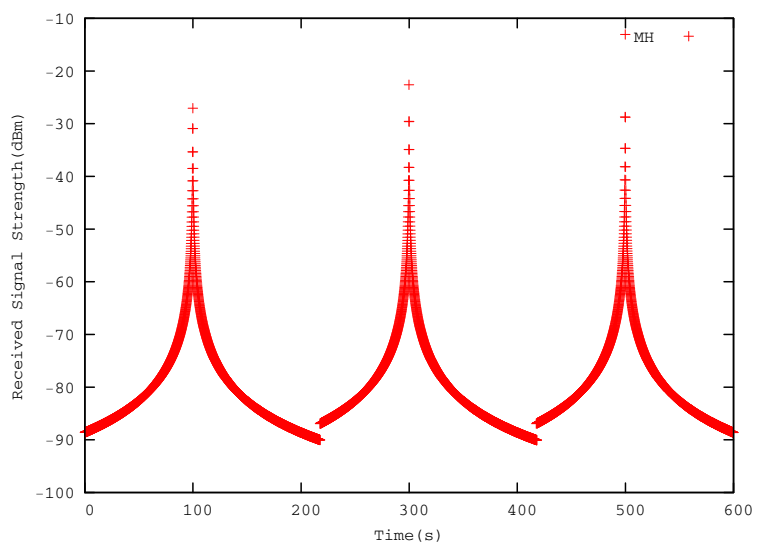

Figure 12. Received Signal Strength

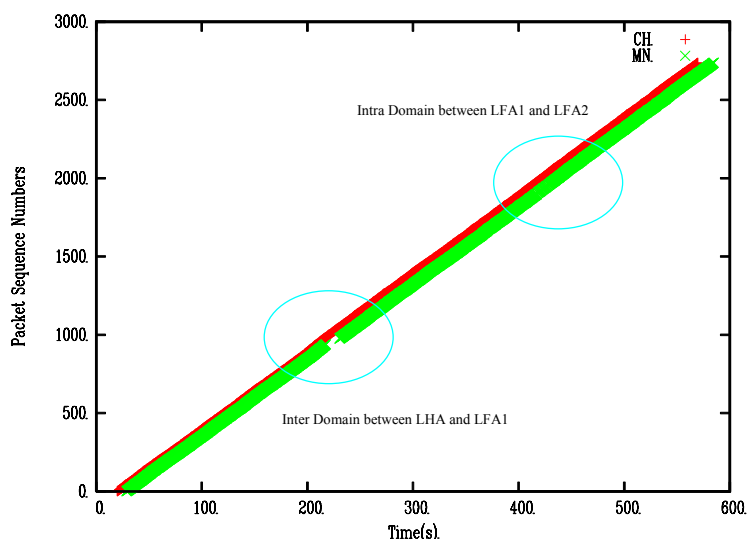

Figure 13. Packet Sequence Numbers in case of no buffering and bicasting

We have tested for many different scenarios and parameters in order to validate the mechanisms: L2 handoff, buffering and bicasting. The simulation scenario is shown in Figure 11. The constant bit rate (CBR) traffic is generated by $\mathrm{CN}$. The destination of traffic is $\mathrm{MN}$. The $\mathrm{MN}$ is located at point of $(0,100,0)$. At 1.0 second, MN start moving to point of $(600,100,0)$ with speed of 1 meter per second. The antenna of MN is Omni antenna with gain of $0 \mathrm{dBi}$. The wireless channels of LHA, LFA1 and LFA2 are 1, 6 and 11 respectively. The cell search threshold, bicasting threshold and buffering threshold are $-90 \mathrm{dBm},-89.5 \mathrm{dBm}$ and 89.5 $\mathrm{dBm}$, respectively. The radio propagation model is free space. The received signal strength is shown in Figure 12.. It could be seen that the result follows to the theory. Figures 13,14, 15, and 16 show the received packet sequence numbers. The MN line presents the MN's received packet sequence numbers. The $\mathrm{CN}$ illustrates CN's sent packet sequence numbers. Figure 14 shows the zoom of the Figure 13 in the duration of intra domain handoff. It could be seen from the figures the inter domain handoff is longer than intra domain handoff since the registration of inter domain handoff is between $\mathrm{MN}$ and $\mathrm{LG}$, whereas the registration of intra domain is between MN and LFA. This is because registration of inter domain handoff needs to communication with LHA; in other words, $t$ du while registration of intra domain handoff communicates between the MN and the LG. Figures 15 and 16 
show the received packet sequence numbers when utilizing bicasting mechanism and buffering mechanism. It could be seen that both mechanisms could reduce lost packets. The average loss rate without bicasting mechanism and buffering mechanism is 1.85 percent. The average loss rate for using bicasting mechanism is 1.71 percent. The average of loss rate for using buffering mechanism is 1.67 percent. The average loss rate for using both bicasting and buffering is 1.63 percent. These could summarize that buffering and bicasting mechanisms could moderately reduce number of lost packets. However, from many experiments have been tested, this summarization depends on many parameters such as cell search threshold, bicasting threshold, buffering threshold, types of scanning used by L2 handoff, etc.

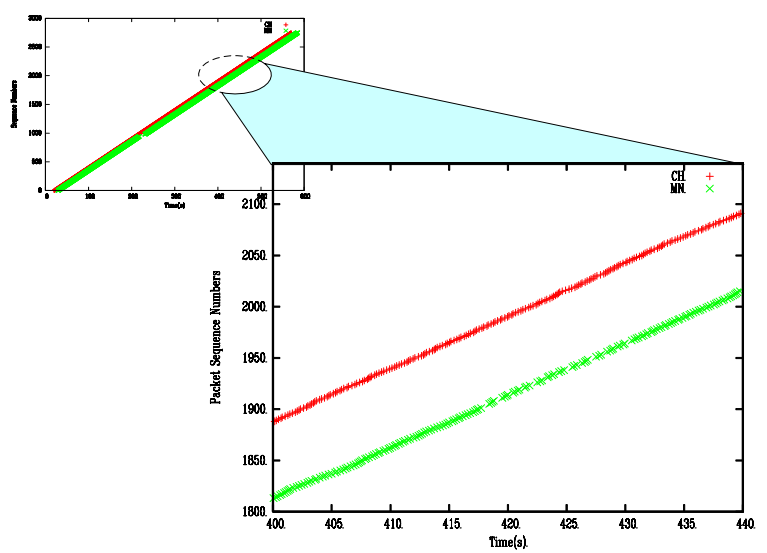

Figure 14. Packet Sequence Numbers in case of no buffering and bicasting mechanisms

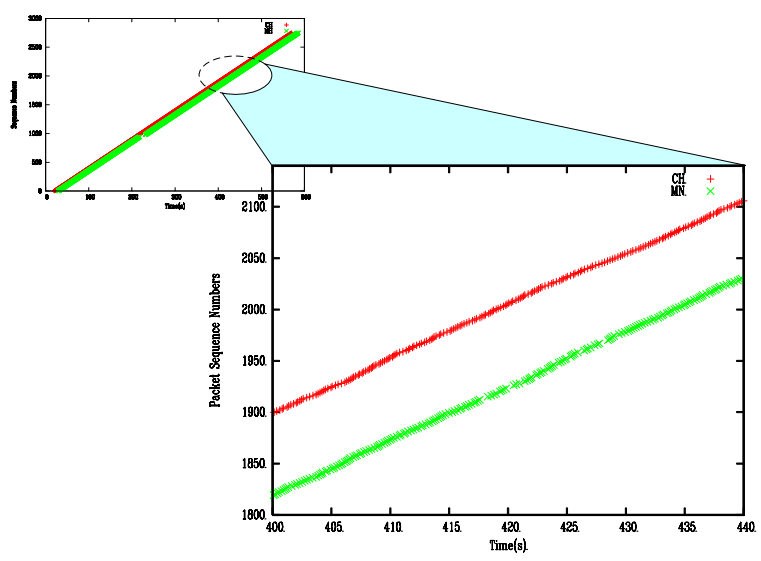

Figure 15. Packet Sequence Numbers in case of using bicasting mechanism

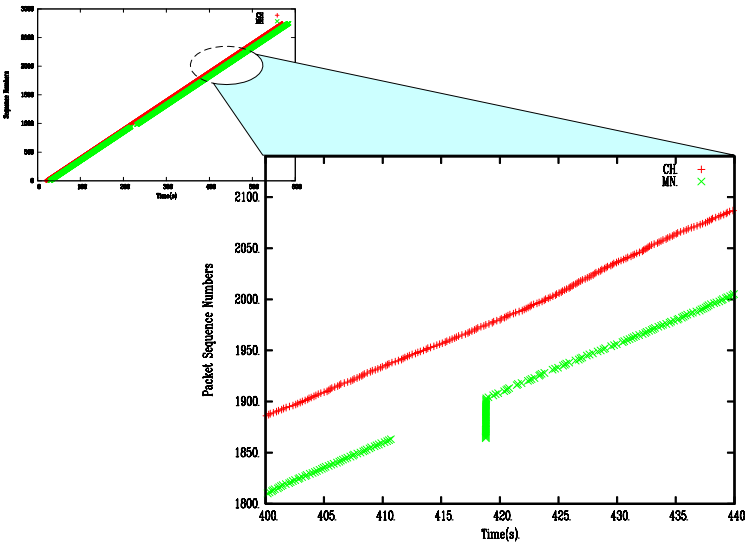

Figure 16. Packet Sequence Numbers in case of using buffering mechanism

\subsection{Multiple Interfaces with Omni and direc- tional Antenna}

In this section, we validate the extended simulator in the case of multiple interfaces and bidirectional antennas. The antenna of LFA is Omni directional. MN has 2 interfaces. Each interface is attached a bi-directional antenna. The pattern of direction is shown in Figure 17 [25]. The gain of the antenna is $14 \mathrm{dBi}$. The angle of antennas is 30 degree to the earth. The $\mathrm{MN}$ is located at $(0,100,0)$. The location of the LFA is $(200,100,10)$. The MN starts moving from the origin to the point of $(400,100,0)$. The received signal strength is shown in Figure 18.. It could be seen that the bidirectional antennas could significantly expand communication range. For example, from the figure, at cell search threshold of $-89 \mathrm{dBm}$, the communication range of omni antenna is about 100 meters in radius while communication of bidirectional antenna is about 160 meters in radius.

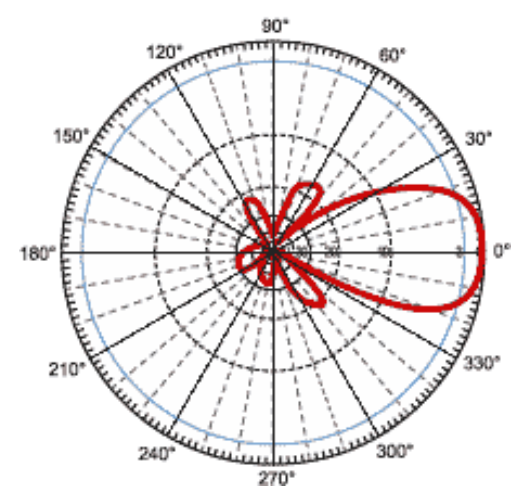

Figure 17. Antenna Radiation Pattern 


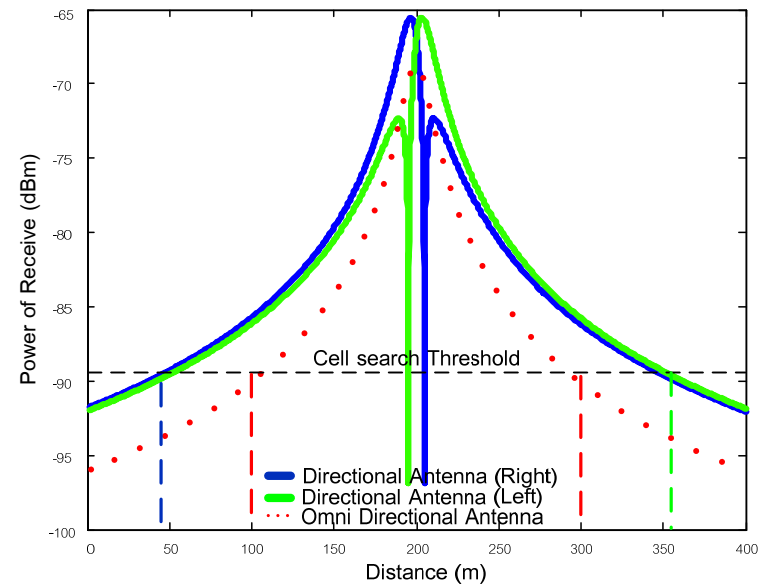

Figure 18. Received Signal Strength for Omni and Directional Antenna

\section{CONCLUSION}

In this paper, we have designed and implemented a generic Micro Mobile MPLS framework for NS-2. The extended NS-2 supports most important mechanisms of Micro Mobile MPLS which is based on IEEE 802.11. The mechanisms are multi-channels, directional antennas, multiple interfaces, bicasting mechanism, and buffering mechanism, pre-established LSP mechanism. Data delivery between LHA and LG could be either tunneling or pure Mobile MPLS. Similarly, data delivery between LG and LFA could be either tunneling or pure Mobile MPLS. The simulation validation shows that the extended simulator follows to the theory. The simulation results show that the bicasting mechanisms, and buffering mechanism could slightly reduce handoff losses. However, these results still depend on many parameters; as examples, cell search threshold, L2-trigger threshold, MN speed. The effects of these parameters will be performed in future. In addition, the simulation results show that the communication range could be extended by using bi-directional antennas.

There are still some important mechanisms needed to be extended; as examples, DS-TE support implemented in [26], multicasting intra- LFA handoff and IEEE 802.11e.

\section{ACKNOWLEDGMENTS}

Our thanks to ACM SIGCHI for allowing us to modify templates they had developed.

\section{REFERENCES}

[1] C. E. Perkins, "Mobile IP," IEEE Communication Magazine, vol. 40, issue 5, pp. 66-82, 2002.

[2] R. Langar, N. Bouabdallah, and S. Tohme, "Handoff support for mobility in future wireless MPLS networks: a Proposal and Analysis," IEEE Wireless Communications and Networking Conference (WCNC), pp. 556-561, 2006.

[3] P. V. Estrela, T. M. Vazao, and M. S. Nunes, "Design and evaluation of eTIMIP - an overlay micro-mobility architecture based on TIMIP," International Conference on Wireless and Mobile Communications (ICWMC'06), 2006.
[4] R. Langar, G. L. Grand, and S. Tohme, "Micro Mobile MPLS in next generation wireless access networks," Proceedings 9th CDMA International Conference (CIC), 2004.

[5] The network simulator - NS-2. http://www.isi.edu/nsnam/ns/.

[6] OPNET Technologies, Inc. http://www.opnet.com/.

[7] Columbia IP micro-mobility software. http://comet.columbia.edu/micromobility/.

[8] The MPLS network simulator. http://flower.ce.cnu.ac.kr/ fog $1 / \mathrm{mns} /$.

[9] G. Ahn and W. Chun, "Design and Implementation of MPLS Network Simulator Supporting LDP and CR-LDP," Proceedings IEEE International Conference on Networks (ICON), 2000.

[10] G. Ahn and W. Chun, "Design and Implementation of MPLS Network Simulator (MNS) supporting QoS," Proceedings 15th IEEE International Conference on Information Networking, 2001.

[11] The Network Simulator: Contributed Code. http://www.cse.hcmut.edu.vn/ ltquan/Documents_Softwares /Network_Simulator/ns-contributed.html.

[12] I. Ganchev, "Enhancement of NS-2 Wireless BS with MPLS to provide a platform for future MuMAcWiN," COST285 2nd MCM, 2003. http://www.cost285.itu.edu.tr/tempodoc/TD_285_03_31_Iva n\%20Ganchev.pdf.

[13] S. Fowler and S. Zeadally, "Fast Handover over MicroMPLS-Based Wireless Networks," Proceeding 11th IEEE Symposium on Computers and Communications (ISCC'06), 2006.

[14] K. Xie, V. W.S. Wong, and V. C.M. Leung, "Support of Micro-Mobility in MPLS-based Wireless Access Networks," IEEE Wireless Communications and Networking (WCNC), 2003.

[15] K. Xie, V. W.S. Wong, and V. C.M. Leung, "Support of Micro-Mobility in MPLS-Based Wireless Access Networks," IEICE Trans. Communications, vol. E88-B, no. 7, 2005.

[16] K. Sethom and H. Afifi, "A new solution for micro-mobility management in next generation networks," Computer and Electrical Engineering, vol. 32, issues 1-3, 2006.

[17] K. Sethom, H. Afifi, and G. Pujolle, "Wireless MPLS: A New Layer 2.5 Micro-mobility Scheme," International Conference on Mobile Computing and Networking, 2004.

[18] O. Horvath, T. Drick, and O. Drobnik, "MTEQ-MPLS: a micro-mobility architecture for dynamic QoS-aware traffic engineering in next generation wireless access networks," Australian Telecommunication Networks and Applications Conference, 2006.

[19] L. Paquereau and B. E. Helvik, "A Module-Based Wireless Node for NS-2," Workshop on ns-2 (WNS2), 2006.

[20] J. Chen, C.-C. Wang, F. C.-D. Tsai, C.-W. Chang, S.-S. Liu, J. Guo, W.-J. Lien, J.-H. Sum, and C.-H. Hung, "The Design and Implementation of WiMAX Module for ns-2 Simulator," Workshop on ns-2 (WNS2), 2006. 
[21] V. Vassiliou, H. L. Owen, D. Barlow, J. Sokol, H.-P. Huth, "M-MPLS: Micromobility-enabled Multiprotocol Label Switching," IEEE International Conference on Communications (ICC), 2003.

[22] Z. Ren, C.-K. Tham, C.-C. Foo, C.-C. Ko, "Integration of mobile IP and multi-protocol label switching," IEEE International Conference on Communications (ICC), 2001.

[23] R. Langar, S. Tohme, and N. Bouabdallah, "Mobility management support and performance analysis for wireless MPLS networks," International Journal of Network Management, 2006.

[24] Q. Y. Xie, H. M. Nguyen, P. Tan, and W. K.G. Seah, "Handover supporting QoS in MPLS-based Hierarchical Mobile IPv6 network," IEEE 58th Vehicular Technology Conference (VTC 2003-Fall), 2003.
[25] "Wireless LAN Antenna", http://www.hyperlinktech.com/multimedia/datasheets/DS_H G2414P.PDF.

[26] RSVP-TE \ns. http://netgroup-serv.iet.unipi.it/rsvp-te_ns/.

[27] "IEEE Std. 802.11, wireless LAN medium access control (MAC) and Physical (PHY) specifications", High Speed Physical Layer Extension in the 2.4 GHz Band, 1999.

[28] Ishwar Ramani, Stefan Savage, "SyncScan: Practical Fast Handoff for 802.11 Infrastructure Networks", Proceedings of the IEEE InfoCom'05, Apr 2005.

[29] P. Chumchu, S. Petnil, N. Rujinark, "IEEE 802.11 Handoff: Analysis and Enhancement”, ITC-CSCC 2006 Conference, 2006.

[30] T. Sanguanwongthong and P. Saengudomlert "MicroMobility MPLS DS-TE for providing QoS VoIP with Fast Handoff support," submitted for publication, 2008. 